\title{
Diagnostic tools in surgically treated patients with infective valve endocarditis
}

\author{
Guenther Kroegh", Khalil Jawad", Piroze Davierwala, Michael Borger, Martin Misfeld \\ Department of Cardiac Surgery, Heart Centre, University of Leipzig, Leipzig, Germany \\ \#These authors contributed equally to this work. \\ Correspondence to: Guenther Kroegh. Department of Cardiac Surgery, Leipzig Heart Centre, Struempelstrasse 39, 04289 Leipzig, Germany. \\ Email: g.krogh.martino@gmail.com.
}

\begin{abstract}
Background: Infective valve endocarditis (IE) is associated with significant mortality and complication rates. The diagnosis impacts not only the prognosis but also the management of the disease. The aim of this article is to show the findings obtained from the implementation of our most important diagnostic tools and discuss our standard diagnostic process for patients with IE who underwent surgical treatment between 1994 and 2017.
\end{abstract}

Methods: Between December 1994 and January 2017 a total of 2,458 patients with IE underwent surgery at our institution. We analyzed clinical, microbiological, echocardiographic, and multi slice computer tomographic (MSCT) features in this group.

Results: The most often isolated involved valve was the aortic valve (59\%). Prosthetic valve endocarditis (PVE) was present in almost one third of all cases. The most common valve failures were mitral valve insufficiency (MI) and aortic valve insufficiency (AI) (65\% and 57\% respectively). Almost one half of the aortic insufficiencies were severe. $63 \%$ of all vegetations assessed by echocardiography were larger than $1 \mathrm{~cm}$. We detected a septic embolism in $44 \%$ of patients and the most common target organ was the spleen (25\%). The most common isolated pathogen was Staphylococcus aureus (26\%) and affected patients had a significant correlation with in-hospital mortality $(\mathrm{P}=0.004)$.

Conclusions: Echocardiography offers valuable information and is the most important diagnostic method in patients with IE. Preoperative MSCT contributes to the entire perioperative decision-making process. Microbiological diagnosis is mandatory in choosing and adjusting antibiotic therapy and also has prognostic value.

Keywords: Cardiac surgery; infective valve endocarditis (IE); diagnostic tools

Submitted Jun 15, 2019. Accepted for publication Oct 28, 2019.

doi: $10.21037 /$ acs.2019.10.06

View this article at: http://dx.doi.org/10.21037/acs.2019.10.06

\section{Introduction}

Infective valve endocarditis (IE) is associated with high mortality and complication rates $(1,2)$. Its long treatment and in some cases, the management of complications such as neurologic dysfunction or large septic spleen embolism followed by a splenectomy, has significant costs to the healthcare system (3).

Early diagnosis and treatment can prevent the most negative consequences (4), but prompt diagnosis remains a challenge, mainly because of the difficulties in identifying the disease due to the wide and sometimes unspecific clinical presentations. This affects the time in which a patient is referred to appropriate specialists to treat the disease, causing delays in treatment, which is often associated with advanced infection and higher mortality and morbidity $(4,5)$.

Uncontrolled local infection, large vegetations with high risk of embolism, and heart valve failure are the main indications for surgery (6). In such cases of advanced disease, it is essential to thoroughly investigate patients 


\begin{tabular}{|ll}
\hline Table 1 Patients' characteristics & \\
\hline Characteristics & $\mathrm{N}[\%]$ \\
\hline Total number of patients & 2,458 \\
\hline Age (years \pm SD) & $62 \pm 14$ \\
\hline Women (\%) & $732[30]$ \\
\hline BMI (kg/m ${ }^{2} \pm$ SD) & $26.7 \pm 5$ \\
\hline Arterial hypertension & $1,606[65]$ \\
\hline Diabetes mellitus-type 2 & $795[32]$ \\
\hline Hypercholesterolemia & $737[30]$ \\
\hline Renal Insufficiency & $858[34]$ \\
\hline Dialysis & $160[7]$ \\
\hline PAD & $268[11]$ \\
\hline COPD & $277[11]$ \\
\hline CAD any stenosis & $651[26]$ \\
\hline Septic shock & $247[10]$ \\
\hline NYHA Class III or IV & $1,251[50]$ \\
\hline Reoperation & $790[32]$ \\
\hline Logistic ES (\% \pm SD) & $21.2 \pm 21.6$ \\
\hline Mean LVEF (\% \pm SD) & $55 \pm 12$ \\
\hline BMI indicates body mass & \\
\hline
\end{tabular}

$\mathrm{BMI}$ indicates body mass index; $\mathrm{PAD}$, peripheral artery disease; COPD, chronic obstructive pulmonary disease; CAD, coronary artery disease; NYHA, New York Heart Association; ES, EuroSCORE; LVEF, left ventricle ejection fraction. Categorical variables are provided as percentages. Continuous variables are provided as mean \pm standard deviation (SD).

with a variety of diagnostic tools; this full evaluation will directly impact decision making and management of these complicated patients and afford valuable prognostic information.

This article aims to present findings from our implementation of important diagnostic tools and discuss the diagnostic process for patients with IE who underwent surgical treatment in our institution between 1994 and 2017.

\section{Methods}

Between December 1994 and January 2017 a total of 2,458 consecutive patients with IE underwent surgery at our institution. IE was defined according to the modified Duke criteria (7) and the indication for surgery was guided by the European Society of Cardiology (ESC) guidelines for infective Endocarditis (6). For this single-center retrospective study, we analyzed clinical, microbiological, echocardiographic, and multi slice computer tomographic (MSCT) features using IBM ${ }^{\mathrm{TM}}$ SPSS Statistics Version 20.0.0.

The differences were tested using a Chi square test as appropriate for binomial/multinomial data. A P value of $<0.05$ was considered statistically significant.

\section{Results}

Over the study period a total of 2,458 patients with IE underwent surgical treatment in our institution. The mean age was 62 years old and 30\% were women. The mean left ventricle ejection fraction (LVEF) was $55 \%$; in one third of the cases it was less than $50 \%$ and in almost $10 \%$ of cases it was less than $30 \%$. Patient characteristics are described in Table 1.

Left sided IE was more common than right sided $(2,261$ versus 120 patients). The most often isolated involved valve was the aortic valve (59\% of all isolated valve IE). Two or more infected valves were present in $19 \%$ of the patients. Prosthetic valve IE (PVE) was present in about $28 \%$ of all cases. Aortic valve prostheses were the most commonly infected (69\% of all PVE).

Concomitant cardiac surgery was done in $44 \%$ of patients-most commonly, coronary artery bypass graft surgery (CABG, 407 patients). A group of 576 patients underwent surgery of the aortic root.

The most common valve failures were mitral insufficiency (MI) and aortic insufficiency (AI) $(65 \%$ and $57 \%$, respectively). Importantly, $48 \%$ of all aortic and $35 \%$ of all mitral valve insufficiencies were severe (grade 3 or 4 ). There was no significant difference in the inhospital mortality for patients with severe aortic or mitral insufficiency $(5.4 \%, \mathrm{~N}=132$ for AI and 3.4\%, N=84 for MI) versus the group with no or mild insufficiency ( $43 \%$, $\mathrm{N}=1,060$ for $\mathrm{AI}$ and $35 \%, \mathrm{~N}=869$ for $\mathrm{MI}$ ) ( $\mathrm{P}$ values $>0.05$ ).

The original infection focus could be identified in $51 \%$ of patients. The most common origin of IE was a dermatological focus or wound infection.

The mean length of the vegetation was $0.8 \mathrm{~cm}$ and $62.8 \%(\mathrm{~N}=1,401)$ of all vegetations assessed with echocardiography $(\mathrm{N}=2,230)$ were over $1 \mathrm{~cm}$. Six patients had a vegetation larger than $3 \mathrm{~cm}$. Patients with vegetations $\geq 1 \mathrm{~cm}$ had significantly more systemic embolization $(\mathrm{P}<0.001)$.

Septic embolization was common affect 1,081 patients 
(44\%), the most common site of embolization was the spleen, followed by the brain. Almost one third of the patients had preoperative neurological dysfunction, which was significantly associated with brain embolization $(\mathrm{P}<0.001)$.

A positive blood culture was seen in 1,939 patients (79\%), the most common isolated bacteria was Staphylococcus aureus. The in-hospital mortality in patients with Staphylococcus aureus was significantly higher than in the group affected by other pathogens ( $18.2 \%$ vs. $13.4 \%, \mathrm{P}=0.004)$.

\section{Discussion}

Once a patient with IE is referred to our department to undergo surgery, a 12-lead electrocardiogram, complete routine laboratory tests, a Doppler of the carotid arteries, and a coronary angiography are performed as part of the standard tests ordered for every cardiac surgery patient in our institution. In IE patients, invasive diagnostic angiography is almost always done per guidelines (8). Emergency surgery cases and the few very large $(>3 \mathrm{~cm})$ and mobile aortic vegetations are the only exceptions. For these cases we use a MSCT to assess the coronary status as recommended (8).

Diagnostic and pre-operative workup is completed according to the endocarditis team, typically, with new blood cultures on admission, a transesophageal echocardiography (TOE) and a full-body MSCT. With this information, we decide the timing of surgery and the makeup of surgical team for each operation. For patients with a prosthetic valve or a device and no clear standard images, we do a positron emission tomography (PET)-CT to improve the accuracy of the diagnosis.

The complexity of endocarditis patients with multiorgan system impairment, requiring radiologic evaluation, and complex antibiotics therapies makes a multi-disciplinary team of experts the best approach to managing IE. In previous publications, this approach has demonstrated an improvement in outcomes (9-11). Our institution's endocarditis team consists of a multidisciplinary group of cardiologists, surgeons, anesthetists, radiologists and intensivists who evaluate and treat each patient individually based on their disease.

In the present study, Staphylococcus aureus was the most common pathogen identified in the blood cultures and its presence could also be associated to a higher mortality. These findings are concordant with previous publications (12) that show that the aggressive course of the disease associated with this pathogen, typically with rapid development of complications and a direct impact on mortality.

Persisting positive blood cultures 48-72 hours after starting antibiotics indicate poor prognosis (13). Given this, we take new blood cultures at the admission for every single patient as a standard measure, even though most patients are referred to our institution with a known pathogen in the blood cultures and an established antibiotic therapy for at least the last 3 days.

Echocardiography plays a central role in the modified Duke diagnostic criteria (14). Transthoracic echocardiography (TTE), has a sensitivity of $70 \%$ and $50 \%$ for the diagnosis of vegetations in native and prosthetic valves, respectively. This can be improved by the use of TOE, a method with $96 \%$ and $92 \%$ sensitivity respectively $(15,16)$. Thus, TOE has an important role in small vegetations and prosthetic valves.

As recommended for every patient with IE undergoing surgical therapy (17), an intraoperative TOE is routine in our center. Local complication assessment, especially by aggressive pathogens like Staphylococcus aureus or patients with PVE (the case for one third of patients in our series) is very important in planning surgery. For example, when a local aortic root abscess is diagnosed before the operation, a root replacement can be planned. This was partially represented in the group of 576 patients who underwent aortic root replacement.

As described in previous studies $(14,18)$, left sided IE with severe valve dysfunction is also a predictor of poor outcome identifiable from echocardiography. Our results show that the most common valve failures were from left sided valves $(65 \%$ mitral valve insufficiency and $57 \%$ aortic valve insufficiency). In this context, it is especially important to note that $48 \%$ of all aortic and $35 \%$ of all mitral insufficiencies were severe (grade 3 or 4). Despite these findings, the statistical analysis failed to show significant differences in the in-hospital mortality of the group with severe aortic or mitral insufficiency versus the group with no or mild insufficiency $(\mathrm{P}>0.05)$. This may be because of the small number of patients in the subgroups.

Determination of LVEF using echocardiography is important for prognosis $(15,16)$. The mean LVEF was $55 \%$ and in one third of all patients it was $<50 \%$. Nine percent $(\mathrm{N}=221)$ of patients had a LVEF $<30 \%$. This was associated with higher in-hospital mortality $(\mathrm{P}<0.001)$.

As described above, an important factor in the risk of embolization and in the indication for surgery is the length 


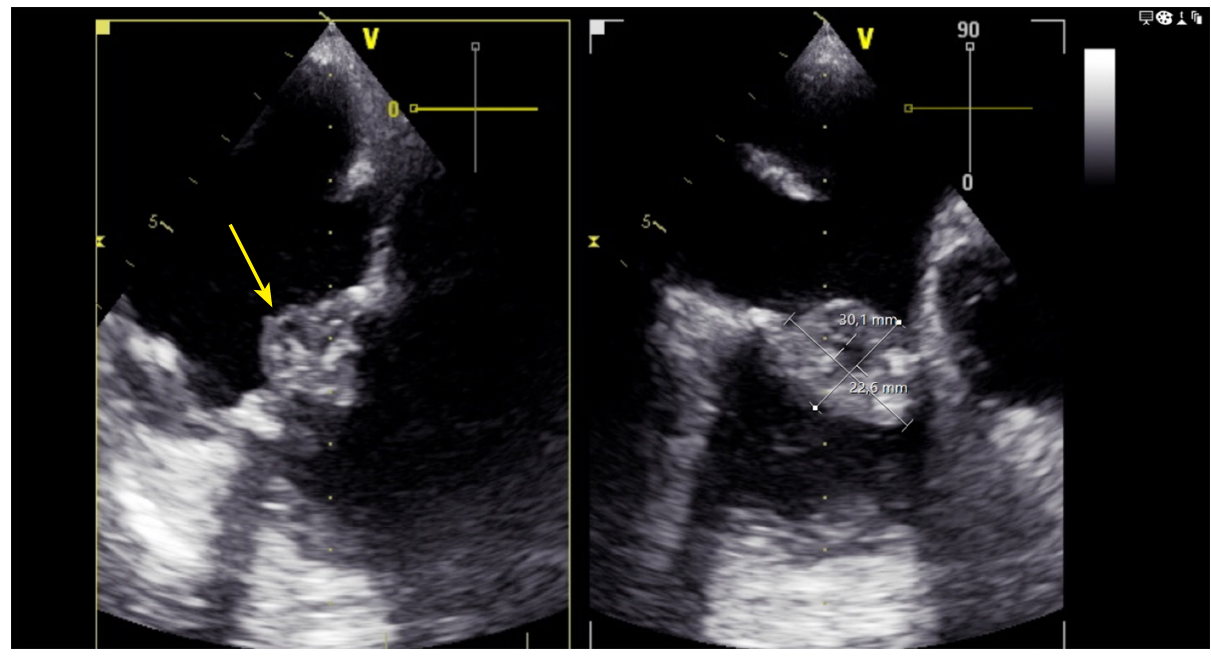

Figure 1 Echocardiography: large vegetation on tricuspid valve (yellow arrow).
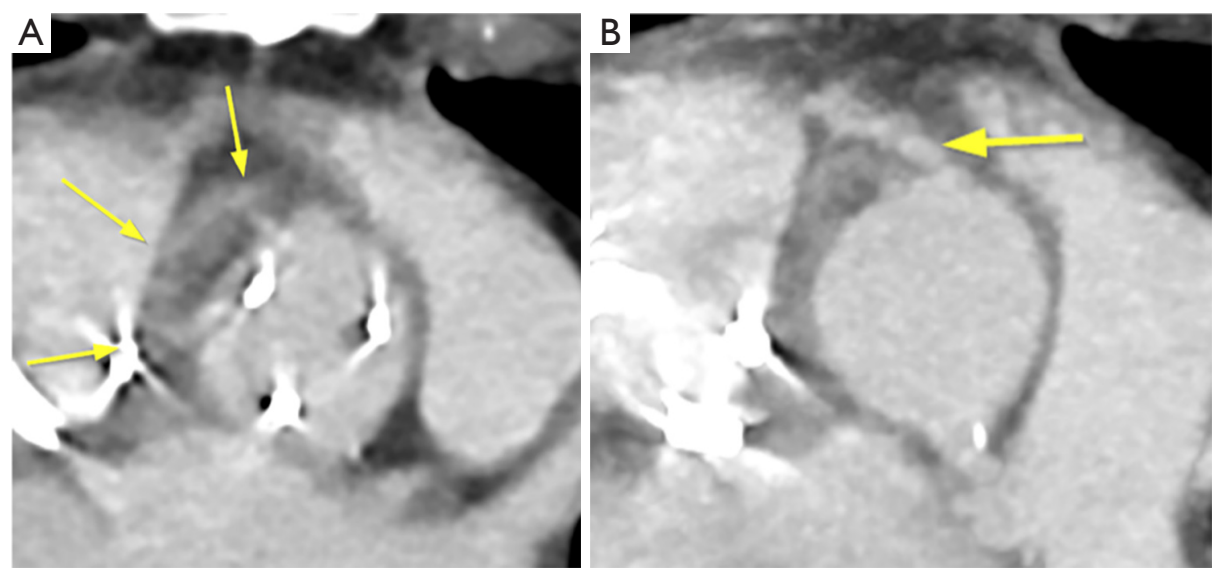

Figure 2 MSCT: root abscess. (A) Yellow arrows indicates aortic root Abscess. (B) Yellow arrow indicates right coronary artery.

of the vegetations $(13,19)$. In this study, the mean length of vegetations in our sample was $0.8 \mathrm{~cm} .63 \%$ of all vegetations examined by echocardiography were over $1 \mathrm{~cm}$ and only 6 patients had a vegetation larger than $3 \mathrm{~cm}$. Patients with vegetations of $\geq 1 \mathrm{~cm}$ had a significantly greater rate of embolization $(\mathrm{P}<0.001)$-this result was in keeping with other published data (17) (Figure 1).

The preoperative complete body MSCT is also a fundamental part of our standard diagnostic methods. It is useful in describing the anatomy and detecting local complications like abscesses, pseudo aneurysms and fistulae (20), which are very important factors to consider when deciding on the best surgical strategy (Figure 2,3). Abscesses and infarcts of target organs, such as the spleen and the brain, can also be assessed in the MSCT images. In the case of a relevant septic embolism in the spleen, a splenectomy is performed subsequently by our abdominal surgeons during a separate surgery. This reinforces the important concept of multidisciplinary team management for these patients (Figure 4).

We determined that the septic embolism rate in our patients was $44 \%(\mathrm{~N}=1,081)$ of the whole group and the most common sites were the spleen and the brain, which was also consistent with previous studies (21). If the patient presents a septic embolism in the brain, the MSCT helps detect any important hemorrhagic transformation, which would delay the time of the surgery as recommended $(22,23)$. In this study, almost one third of the patients 


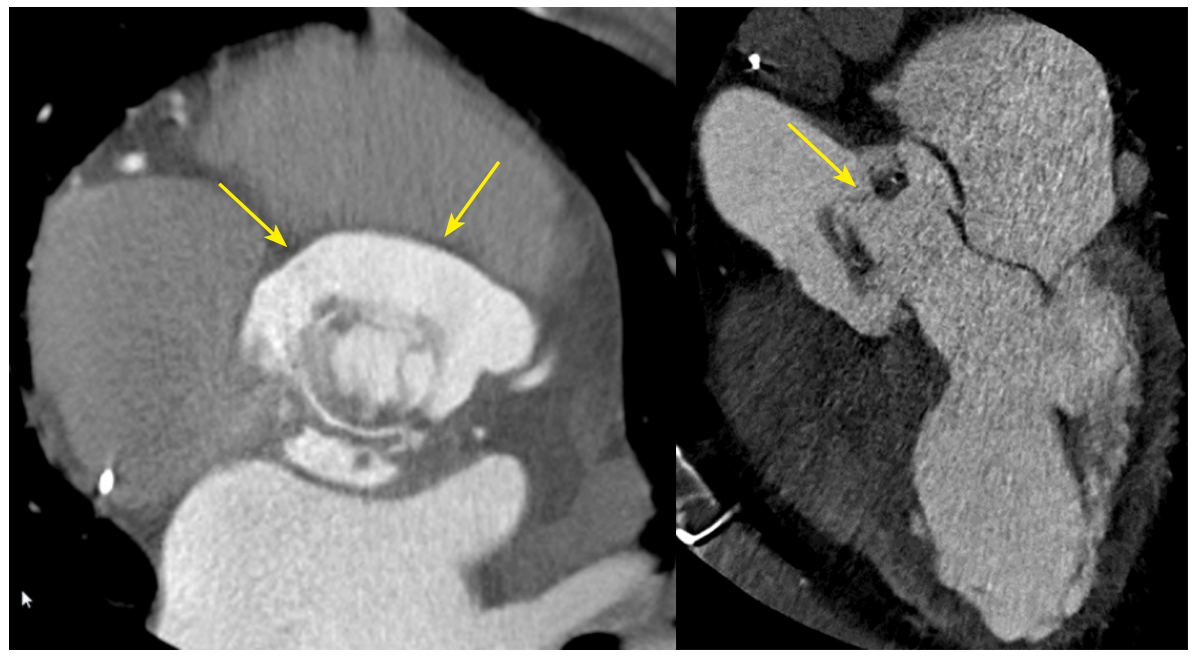

Figure 3 MSCT: pseudoaneurysm and fistulae (yellow arrows).

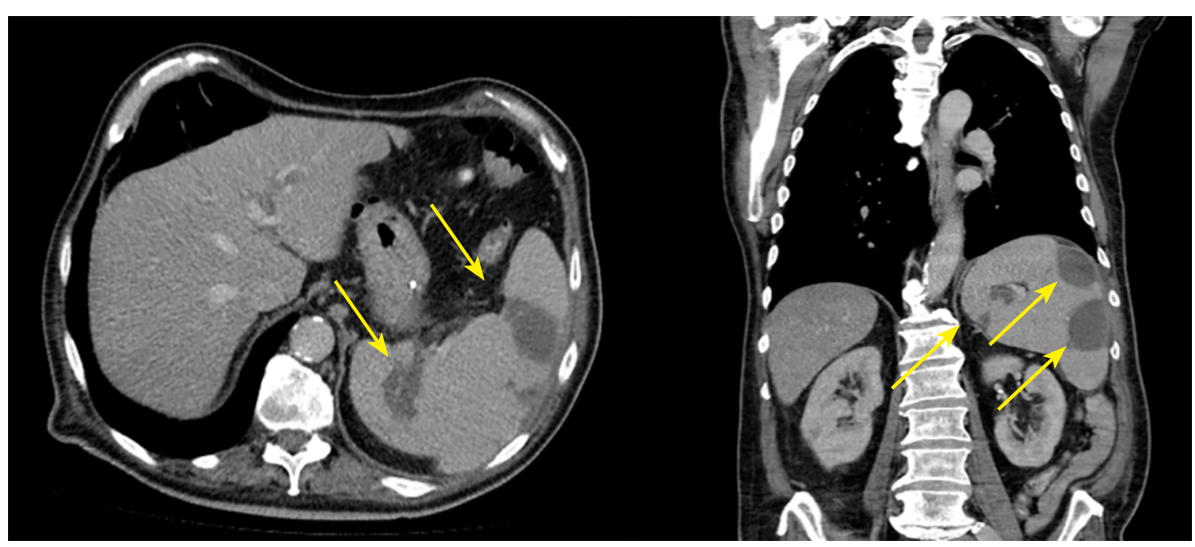

Figure 4 MSCT: multiple spleen embolisms (yellow arrows).

had preoperative neurological dysfunction, which was significantly associated with brain embolization $(\mathrm{P}<0.001)$ (Figure 5).

The long study period with a sample of 2,458 patients could be seen as an advantage, however, recent changes such as the creation of an endocarditis team, improvement of diagnostic tests, and surgical experience, the presented results could have significant variations among the patients who had their surgery at the beginning and at the end of the analyzed period, which is an indication for future studies in our institution.

This study is also retrospective which means there is a risk of confounding and bias is more likely to be present when compared to prospective studies.

As a single center experience, it is difficult to reproduce the results of our study given procedural and management differences which are often unique to each centre. Multicentre, prospective studies are indicated to validate results with regards to management of IE.

\section{Conclusions}

Echocardiography is the most important diagnostic tool used for our patients with IE given the valuable diagnostic and prognostic information it provides. Preoperative CT contributes to the entire decision-making process, before, during and after surgery. Blood cultures are mandatory in choosing and adjusting the antibiotic therapy. Coronary angiography can be safely done in most cases despite vegetations on the aortic valve. We use PET CT only 


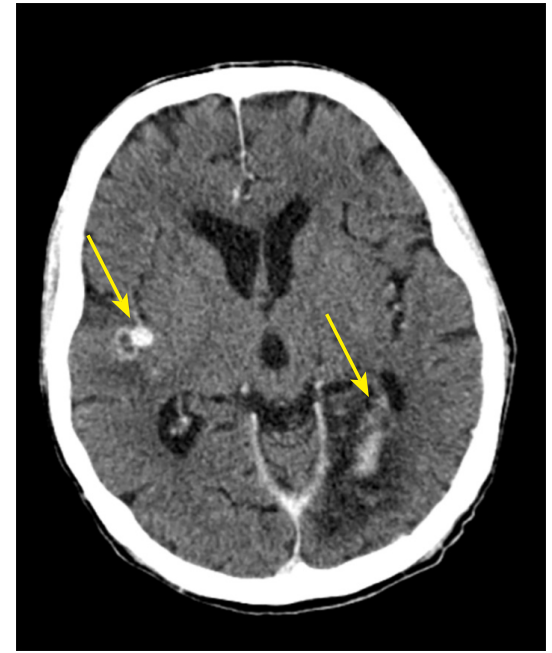

Figure 5 MSCT: brain abscess and embolism (yellow arrows).

in suspected prosthesis or device-related IE and only in addition to our standard diagnostic tools when these are not congruent with the clinical picture. A multidisciplinary evaluation and management by an endocarditis team is mandatory for these complex patients.

\section{Acknowledgments}

None.

\section{Footnote}

Conflicts of Interest: The authors have no conflicts of interest to declare.

\section{References}

1. Leone S, Ravasio V, Durante-Mangoni E, et al. Epidemiology, characteristics, and outcome of infective endocarditis in Italy: the Italian Study on Endocarditis. Infection 2012;40:527-35.

2. Murdoch DR, Corey GR, Hoen B, et al. Clinical presentation, etiology, and out- come of infective endocarditis in the 21st century: the International Collaboration on Endocarditis-Prospective Cohort Study. Arch Intern Med 2009;169:463-73.

3. Sunder S, Grammatico-Guillon L, Baron S, et al. Clinical and economic outcomes of infective endocarditis. Infect Dis (Lond) 2015;47:80-7.

4. Aksoy O, Sexton DJ, Wang A, et al. Early surgery in patients with infective endocarditis: a propensity score analysis. Clin Infect Dis 2007;44:364-72.

5. Middlemost S, Wisenbaugh T, Meyerowitz C, et al. A case for early surgery in native left-sided endocarditis complicated by heart failure: results in 203 patients. J Am Coll Cardiol 1991;18:663-7.

6. Habib G, Lancellotti P, Antunes MJ, et al. 2015 ESC Guidelines for the management of infective endocarditis: The Task Force for the Management of Infective Endocarditis of the European Society of Cardiology (ESC). Endorsed by: European Association for Cardio-Thoracic Surgery (EACTS), the European Association of Nuclear Medicine (EANM). Eur Heart J 2015;36:3075-128.

7. Li JS, Sexton DJ, Mick N, et al. Proposed modifications to the Duke criteria for the diagnosis of infective endocarditis. Clin Infect Dis 2000;30:633-8.

8. Vahanian A, Alfieri O, Andreotti F, et al. Guidelines on the management of valvular heart disease (version 2012). Eur Heart J 2012;33:2451-96.

9. Chirillo F, Scotton P, Rocco F, et al. Impact of a multidisciplinary management strategy on the outcome of patients with native valve infective endocarditis. Am J Cardiol 2013;112:1171-6.

10. Lancellotti P, Rosenhek R, Pibarot P, et al. ESC Working Group on Valvular Heart Disease position paper-heart valve clinics: organization, struc- ture, and experiences. Eur Heart J 2013;34:1597-606.

11. Botelho-Nevers E, Thuny F, Casalta JP, et al. Dramatic reduction in infective endocarditis-related mortality with a management-based approach. Arch Intern Med 2009;169:1290-8.

12. Chirouze C, Cabell CH, Fowler VG Jr, et al. Prognostic factors in 61 cases of Staphylococcus aureus prosthetic valve infective endocarditis from the International Collaboration on Endocarditis merged database. Clin Infect Dis 2004;38:1323-7.

13. Lopez J, Sevilla T, Vilacosta I, et al. Prognostic role of persistent positive blood cultures after initiation of antibiotic therapy in left-sided infective endocarditis. Eur Heart J 2013;34:1749-54.

14. Chu VH, Cabell CH, Benjamin DK Jr, et al. Early predictors of in-hospital death in infective endocarditis. Circulation 2004;109:1745-9.

15. Habib G, Badano L, Tribouilloy C, et al.

Recommendations for the practice of echocardiography in infective endocarditis. Eur J Echocardiogr 2010;11:202-19.

16. Mugge A, Daniel WG, Frank G, et al. Echocardiography in infective endocarditis: reassessment of prognostic 
implications of vegetation size determined by the transthoracic and the transesophageal approach. J Am Coll Cardiol 1989;14:631-8.

17. Shapira Y, Weisenberg DE, Vaturi M, et al. The impact of intraoperative transesophageal echocardiography in infective endocarditis. Isr Med Assoc J 2007;9:299-302.

18. San Roman JA, Lopez J, Vilacosta I, et al. Prognostic stratification of patients with left-sided endocarditis determined at admission. Am J Med 2007;120:369-7.

19. Kang DH, Kim YJ, Kim SH, et al. Early surgery versus conventional treatment for infective endocarditis. N Engl J Med 2012;366:2466-73.

20. Feuchtner GM, Stolzmann P, Dichtl W, et al. Multislice computed tomography in infective endocarditis: comparison with transesophageal echocardiography and in- traoperative findings. J Am Coll Cardiol 2009;53:436-44.

21. Thuny F, Di Salvo G, Belliard O, et al. Risk of embolism and death in infective endocarditis: prognostic value of echocardiography: a prospective multicenter study. Circulation 2005;112:69-75.

22. Yoshioka D, Sakaguchi T, Yamauchi T, et al. Impact of early surgical treatment on postoperative neurologic outcome for active infective endocarditis compli- cated by cerebral infarction. Ann Thorac Surg 2012;94:489-95.

23. Eishi K, Kawazoe K, Kuriyama Y, et al. Surgical management of infective endocarditis associated with cerebral complications. Multi- center retrospective study in Japan. J Thorac Cardiovasc Surg 1995;110:1745-55.

Cite this article as: Kroegh G, Jawad K, Davierwala P, Borger M, Misfeld M. Diagnostic tools in surgically treated patients with infective valve endocarditis. Ann Cardiothorac Surg 2019;8(6):654-660. doi: 10.21037/acs.2019.10.06 